\title{
Brain development and scholastic achievement in the Education Quality Measurement System tests in Chilean school-aged children
}

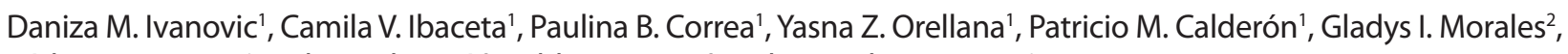
Bárbara D. Leyton ${ }^{1}$, Atilio F. Almagià̀ ${ }^{2}$ Pablo A. Lizana ${ }^{3}$ and Raquel A. Burrows ${ }^{1}$

BACKGROUND: Head circumference $(\mathrm{HC})$, the anthropometric index of both brain development and nutritional background, has been described to be significantly associated with scholastic achievement (SA). The aim of this study was to determine the impact of nutritional background and current nutritional status parameters on SA in the Education Quality Measurement System (SIMCE) tests.

METHODS: A representative sample of 33 schools was randomly chosen in the Metropolitan Region of Chile. The sample consisted of 1,353 school-aged children of both sexes, from the fifth grade of elementary school and from the first grade of high school who in 2009 took the SIMCE tests. Nutritional status was assessed through anthropometric parameters. Brain development was measured through the $\mathrm{HC}$ expressed as HC-for-age Z-score (Z-HC).

RESULTS: Students with $Z-H C<-2$ SD and $>2$ SD obtained low and high SA, respectively, both in the language and the mathematics tests $(P<0.001)$. In general, in both grades, those students with $Z-H C \geq 0$ SD increase more than double the probability to obtain language and mathematics SA scores $\geq$ the median $(P<0.0001)$.

CONCLUSION: We confirm the hypothesis that $\mathrm{HC}$ is the most relevant physical index associated with SA; therefore, children with the lowest scores in the SIMCE tests probably have lower brain development.

$S_{b}^{c}$ everal authors have emphasized the significant relation between brain development and cognition in children $(1,2)$. Head circumference (HC) has been defined as an anthropometric indicator of both nutritional background and brain development (3). In this context, the findings from several investigations underline that $\mathrm{HC}$ at the age of $1 \mathrm{y}$ rather than that at birth determines psychomotor development (2). Birth $\mathrm{HC}$ and growth in $\mathrm{HC}$ in the first 6 mo were better predictors than birth weight or intelligence (4).

Findings by other authors reveal that poor prenatal and postnatal HC growth results in poor outcomes in terms of the acquisition of cognitive and academic abilities by the child, and this group is followed by those children with prenatal brain compromise but satisfactory postnatal $\mathrm{HC}$ growth (5). Other authors observed that social, neonatal, and developmental factors at $30 \mathrm{mo}$, such as $\mathrm{HC}$ and cognitive development, were independent predictors of educational outcomes at $11 \mathrm{y}$ of age (6).

Low maternal education is associated with slower fetal growth, and this effect appears to be stronger for growth of the head than for growth of other organs (7). Maternal intelligence quotient, home environment, ethnic group, and number of siblings have been described as important predictors of child intelligence quotient. A 1-cm decrease in $\mathrm{HC}$ predicted a 1-point decrease in the Stanford-Binet composite score (8). Mother's educational level, gestational age, and $\mathrm{HC}$ at age $2 \mathrm{y}$ could explain the achievement of appropriate schooling at age 8 y (9).

In a review of the literature published in the past $5 \mathrm{y}$, these studies identified both risk factors (lower birth weight, intraventricular hemorrhage, and low maternal educational level) and protective factors (larger HC, breastfeeding, and higher family income) modulating the development and quality of life of preschool and school-aged children born preterm (10). Small for gestational age at birth and microcephaly at $2 \mathrm{y}$ were independently related to $16-y$ intelligence quotient (11). Findings by other authors suggest that abnormal brain development after perinatal injury or postnatal nutritional deficits are responsible for cognitive deficits in preterm children (12). Preterm birth has long-term effects on cognition, behavior, and future academic achievement primarily as a consequence of global reduction in brain white matter (13). Other studies reveal that breast milk feeding is significantly correlated with intelligence, total brain volume (BV), and total white matter in boys (14). Thus, human brain structure and intelligence can be influenced by early nutrition (15).

Cognition and motor skills may be interrelated with brain structure and behavior (16). For all age and sex groups, it

${ }^{1}$ Institute of Nutrition and Food Technology, University of Chile, Santiago, Chile; ${ }^{2}$ Public Health Department, University of La Frontera, Temuco, Chile; ${ }^{3}$ Laboratory of Physical Anthropology and Human Anatomy, Institute of Biology, Pontificia Universidad Católica de Valparaíso, Valparaiso, Chile. Correspondence: Daniza M. Ivanovic (daniza@inta.uchile.cl) 
appears that BV is positively correlated with intelligence (17). Genes influencing the variability in both cognition and brain plasticity partly drive these associations. Thus, not only does the brain continue to change well into adulthood, but these changes are functionally relevant because they are related to intelligence (18); this has been described as the best predictor of scholastic achievement (SA) (19).

Our previous studies reveal that HC (and not weight or height) is the most relevant physical growth index associated with SA, intellectual ability, and school dropout in Chilean school-aged children (19-22). Microcephalic children (HC-for-age $Z$-score $(Z-H C)<-2 \mathrm{SD})$ exhibit multiple disorders related not only to BV but also to intelligence quotient, $\mathrm{SA}$, and nutritional background, variables that are significantly interrelated (3).

The aim of this study was to determine the impact of (i) the nutritional background and (ii) parameters of current nutritional status on language and mathematics SA in the Education Quality Measurement System (SIMCE) tests administered by the Ministry of Education; this is the first study carried out in Chile using a test with national coverage to measure SA and its association with anthropometric parameters of nutritional status. The purpose was to confirm the hypothesis that independently of grade, school-aged children with the highest scores in the SIMCE tests for language and mathematics have the highest $\mathrm{HC}$ values compared with their peers with medium and low $\mathrm{SA}$ and that $\mathrm{HC}$ is the most relevant anthropometric parameter associated with SA for both language and mathematics.

\section{RESULTS}

Regarding Z-HC, 2.4 and $5.2 \%$ of the sample registered values $<-2$ SD (microcephaly) and $>2$ SD (macrocephaly), respectively. Most of the sample (92.4\%) had a "normal HC" (mean \pm 2 SD); however, $39.6 \%$ exhibited $Z$-HC values between -2 and $<0$ SD and $52.8 \%$ had values between 0 and 2 SD.

A positive and significant association between $Z$-HC and SA in the SIMCE test for both language and mathematics was registered in the total sample. In the language test, most of the children with Z-HC > 2 SD had high SA (54.4\%), whereas those with $Z$-HC $<-2$ SD mainly registered medium (61.9\%) and low SA $(23.8 \% ; P<0.001)$. In the mathematics test, SA was more evident because 63.2 and $69.6 \%$ of children with $Z$-HC $<$ $-2 \mathrm{SD}$ and $>2 \mathrm{SD}$ achieved low and high SA levels, respectively $(P<0.001$; Figure 1$)$. This was independent of school grade and sex.

Figure 2 shows that, in the total sample, height-for-age $Z$-score $(Z-H)$ and language SA were not significantly associated, and this was observed in both school grades and in males, because in females, 42.9 and $58.8 \%$ of those with severe growth failure and tall features registered low and high SA, respectively $(P<0.05)$. In mathematics $S A$, in the total sample, 42.9 and $47 \%$ of children with severe growth failure and tall features exhibit low and high SA, respectively $(P<0.01)$. This was observed independently of sex and only in the first grade of high school because 25.9 and $13 \%$ of children with low and high SA exhibited growth failure $(P<0.01)$. a

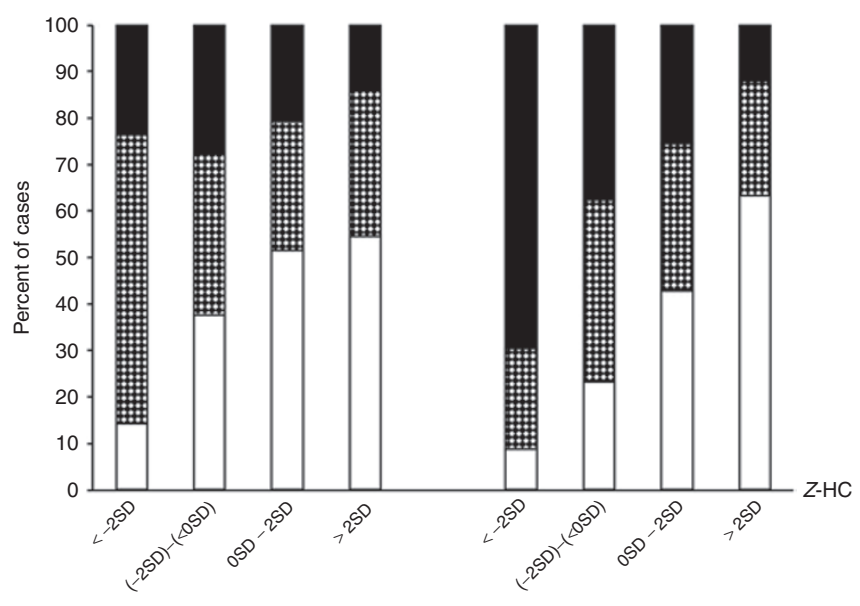

Figure 1. Scholastic achievement (SA) in the 2009 Education Quality Measurement System test of Chilean school-aged children by head circumference-for-age $Z$-score $\left(Z\right.$-HC). (a) Language $\left(\chi_{0}^{2}(6 \mathrm{~d} f)=33.4902\right.$; $P<0.001)$; (b) mathematics $\left(\chi_{0}^{2}(6 \mathrm{~d} f)=80.0188 ; P<0.001\right)$. White columns, high SA; dotted columns, medium SA; and black columns, low SA. df, degrees of freedom.

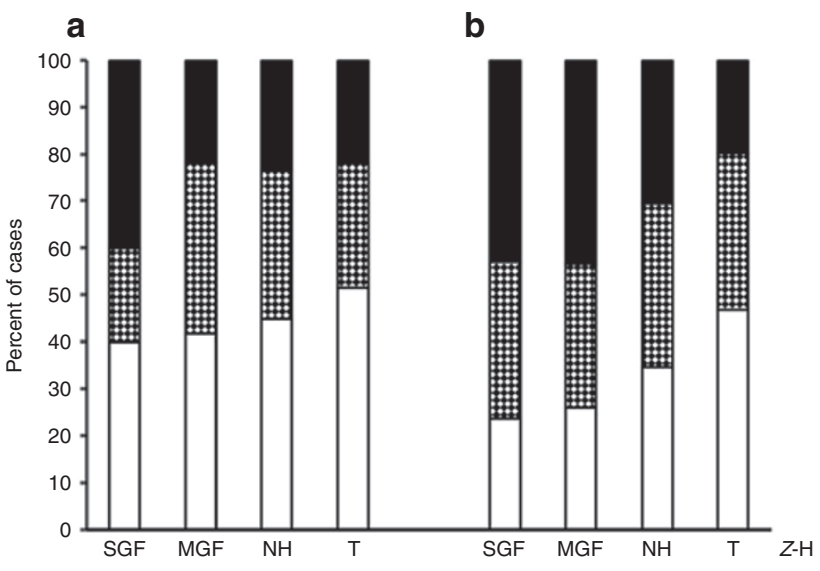

Figure 2. Scholastic achievement (SA) in the 2009 Education Quality Measurement System test of Chilean school-aged children by height-forage $Z$-score $(Z-H)$. (a) Language $\left(\chi_{0}^{2}(6 \mathrm{~d} f)=8.1980 ; P<0.05\right)$; (b) mathematics $\left(\chi_{0}^{2}(6 \mathrm{~d} f)=26.9063 ; P<0.01\right)$. White columns, high SA; dotted columns, medium SA; and black columns, low SA. df, degrees of freedom; MGF, mild growth failure; $\mathrm{NH}$, normal height; SGF, severe growth failure; T, tall.

In the total sample, both in school grades and in males, BMIfor-age $Z$-score (Z-BMI) was not significantly associated with SA in both the language and the mathematics tests (Figure 3 ). In females, only in language SA, 19.3 and $49.3 \%$ of those who obtained low and high SA had normal $Z$-BMI $(P<0.05)$.

Table 1 expresses the scores (mean (SD)) obtained in the language and mathematics SA by nutritional status and grade. In both school grades, children with the highest $Z$-HC values scored significantly higher in the SA tests for both language $(P<0.01)$ and mathematics $(P<0.0001)$. As regards $Z-H$, in both grades, a positive and significant association, although with low significance, was observed only for mathematics SA $(P<0.01)$. According to $Z$-BMI categories, a positive and significant association was observed only for language SA $(P<0.01)$. 


\section{Articles | Ivanovic et al.}

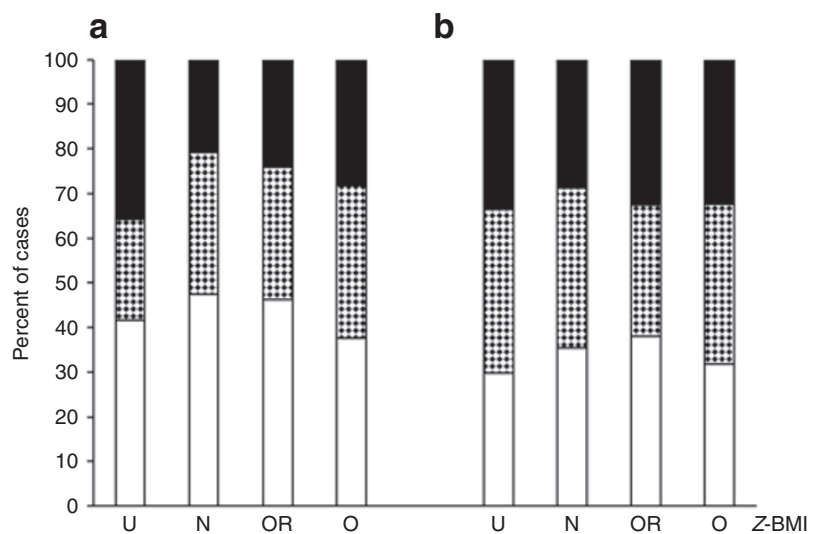

Figure 3. Scholastic achievement (SA) in the 2009 Education Quality Measurement System test of Chilean school-aged children by BMI-for-age $Z$-score (Z-BMI). (a) Language $\left(\chi_{0}^{2}(6 \mathrm{~d} f)=10.3232 ; \mathrm{NS}\right)$; (b) mathematics $\left(\chi_{0}^{2}(6 \mathrm{~d} f)=5.5146\right.$; NS). White columns, high SA; dotted columns, medium SA; and black columns, low SA. N, normal; NS, not significantly different; $\mathrm{O}$, obesity; OR, obesity risk; $U$, underweight.

The logistic regression analysis between language and mathematics SA scores in the SIMCE test (dependent variable), probability-modeled scores $\geq$ median (Md), anthropometric parameters, and sex (independent variables; Table 2) revealed that, in both school grades, Z-HC was the independent variable with the greatest explanatory power in the language and mathematics SA scores. In students from the fifth grade of elementary school, Z-HC was the best predictor for language and mathematics SA scores (odds ratio value $=1.510$ and 2.447 , respectively), which indicates that $Z$-HC scores $\geq 0$ $\mathrm{SD}$ increases the probability of obtaining language and mathematics SA scores $\geq$ Md by $51 \%$ and $244.7 \%$, respectively. Comparable results were observed in students from the first grade of high school because $Z$-HC was also the best predictor for language and mathematics SA scores (odds ratio value $=2.030$ and 2.097, respectively), which indicates that $Z-\mathrm{HC}$ scores $\geq 0$ SD increase the probability of obtaining language and mathematics SA scores $\geq$ Md by 203 and $209.7 \%$, respectively; the male sex appears as a preventive factor (odds ratio value $=0.578$ ) because being males reduced the probability of mathematics SA scores $<$ Md by $42.2 \%$.

\section{DISCUSSION}

The results of this study confirm that HC is the most relevant anthropometric parameter associated significantly with SA in the SIMCE tests and this was observed independently of school grade. Z-H and Z-BMI did not contribute to the explanation of SA. As a consequence, $\mathrm{HC}$, an anthropometric indicator of both nutritional background and brain development, constitutes the most important physical index associated with SA. This is relevant because SA evaluated through the SIMCE tests, the dependent variable of this study, has a national coverage as a parameter of quality-of-education measurement in Chile. Therefore, extrapolating from the results of this study, we may hypothesize that children with suboptimal $\mathrm{HC}$ have some degree of alteration of brain development associated with lowered SA in the SIMCE tests, thus confirming some previous results (3). Sex (males) was a preventive factor for mathematics SA only in students from the first year of high school, probably because they have greater mathematical ability (23).

The nutritional status of Chilean school-aged children has been positively and significantly correlated with SA and intelligence, especially with indicators of past nutrition; however, as previously stated, $\mathrm{HC}$ is the most relevant index associated with intelligence and learning success $(3,19-22)$. Children with suboptimal HC graduating from high school have a high probability of failing the Academic Aptitude Test for university admission (22). The explanatory power of HC on intellectual ability and SA increases significantly from the onset of elementary school until the end of high school; in contrast with this, the explanatory power of body weight and height decreases significantly. Furthermore, educational selectivity correlates with $\mathrm{HC}$ and not with weight and height (21). Findings from other authors confirm that students with intellectual disabilities had smaller total BVs (24).

$\mathrm{HC}$ represents the most sensitive physical index of undernutrition during the first year of life and is strongly related with prenatal parameters, $\mathrm{HC}, \mathrm{BV}$, intelligence, and SA in children at $18 \mathrm{y}$ of age when they graduate from high school (25).

Research with the aim of defining the nutrients that are important for the adequate development of brain structure and function during gestation and early childhood, with the ultimate goal of improving cognitive development, is an important task in terms of educational and future public health policies. Some investigations have underlined the need for adequate supplies of $\omega-3$ fatty acids during pregnancy, but evidence about its effects on birth outcomes is limited, although some authors emphasize that prenatal docosahexaenoic acid supplementation to primigravid women may result in increased birth size in a population where dietary docosahexaenoic acid intakes are low (26).

In this study, most part of the sample had a "normal HC" (mean $\pm 2 \mathrm{SD}$ ), although SIMCE results significantly decreased in school-aged children with $Z$-HC values already immediately below the $0 \mathrm{SD}$ or mean values. This means that $Z$-HC values lower than the mean are associated with an increased incidence of lower SA scores in the SIMCE test and that small differences in $\mathrm{HC}$ could be of considerable importance in the HC-SA interrelationship, a finding in agreement with our previous results $(3,20)$. As in previous studies, we emphasize that a "normal" HC (mean $\pm 2 \mathrm{SD}$ ) is more related to statistical normality, although this may not be so for psychological or educational achievements $(3,20)$. However, the findings of this study should be considered as showing a statistical association and that these do not represent a direct cause-and-effect relationship.

Despite the fact that most children in the sample had a normal HC, children whose $\mathrm{HC}$ was below mean had lower SA. This finding needs more attention and research because in the sample described in this study, the lowered HC is not due to undernutrition at an early age because these schoolaged children had not been affected by it. Another possible explanation for our results is that $\mathrm{HC}$ is a highly heritable biometric trait because in nearly all age groups, the largest part 
Table 1. Language and mathematics SA scores in the SIMCE test by nutritional status and grade

\begin{tabular}{|c|c|c|c|c|}
\hline Nutritional status & \multicolumn{2}{|c|}{ Fifth grade of elementary school } & \multicolumn{2}{|c|}{ First grade of high school } \\
\hline \multicolumn{5}{|l|}{$\mathrm{Z}-\mathrm{HC}$} \\
\hline-2 to $<0 S D$ & $258.6^{\mathrm{a}}(63.5)(n=212)$ & $247.8^{\mathrm{a}}(52.0)(n=210)$ & $273.3^{\mathrm{a}, \mathrm{b}}(45.7)(n=209)$ & $281.3^{b}(50.3)(n=213)$ \\
\hline $0-2 S D$ & $275.5^{\mathrm{b}}(53.3)(n=323)$ & $273.3^{b}(54.3)(n=323)$ & $283.4^{\mathrm{b}}(50.6)(n=268)$ & $300.4^{c}(53.7)(n=267)$ \\
\hline$>2 S D$ & $F=5.08 ; P<0.01$ & $F=12.53 ; P<0.0001$ & $F=4.17 ; P<0.01$ & $F=14.18 ; P<0.0001$ \\
\hline \multicolumn{5}{|l|}{$\mathrm{Z}-\mathrm{H}$} \\
\hline SGF & $258.7(48.2)(n=7)$ & $256.4^{\mathrm{a}, \mathrm{b}}(56.2)(n=8)$ & $262.4(64.2)(n=13)$ & $264.1^{\mathrm{a}, \mathrm{b}}(65.8)(n=13)$ \\
\hline MGF & $256.9(57.8)(n=48)$ & $248.6^{\mathrm{a}}(60.7)(n=48)$ & $273.2(51.0)(n=86)$ & $277.2^{\mathrm{a}}(54.1)(n=86)$ \\
\hline $\mathrm{NH}$ & $268.0(53.5)(n=379)$ & $262.1^{\mathrm{a}}(55.1)(n=376)$ & $279.8(48.0)(n=382)$ & $294.0^{\mathrm{b}}(52.6)(n=382)$ \\
\hline \multicolumn{5}{|c|}{ Current nutritional status } \\
\hline \multicolumn{5}{|l|}{ Z-BMI } \\
\hline U & $261.2(53.0)(n=26)$ & $257.7(49.8)(n=25)$ & $277.4^{\mathrm{a}, \mathrm{b}}(47.4)(n=5)$ & $261.8(56.5)(n=5)$ \\
\hline $\mathrm{N}$ & $271.7(52.9)(n=255)$ & $266.3(54.9)(n=256)$ & $283.4^{\mathrm{b}}(48.3)(n=292)$ & $293.7(52.3)(n=291)$ \\
\hline OR & $269.8(54.0)(n=166)$ & $264.7(56.0)(n=166)$ & $278.4^{\mathrm{b}}(49.0)(n=152)$ & $295.5(54.9)(n=156)$ \\
\hline \multirow[t]{2}{*}{$\mathrm{O}$} & $265.3(56.8)(n=117)$ & $261.5(54.9)(n=117)$ & $259.5^{\mathrm{a}}(49.3)(n=76)$ & $279.9(53.5)(n=76)$ \\
\hline & $F=0.57 ; \mathrm{NS}$ & $F=0.34 ; \mathrm{NS}$ & $F=4.83 ; P<0.01$ & $F=2.15 ; \mathrm{NS}$ \\
\hline
\end{tabular}

Scores are expressed as mean (SD). The number of cases in each group is indicated between parentheses. Means with the same letter are not significantly different at the 0.05 level based on Bonferroni's test.

MGF, mild growth failure; N, normal; O, obesity; OR, obesity risk; SGF, severe growth failure; SIMCE, scholastic achievement in the Education Quality Measurement System; T, tall; U, underweight; W, well nourished; Z-BMI, BMI-for-age Z-score; Z-H, height-for-age Z-score; Z-HC, head circumference-for-age Z-score.

of the variation in $\mathrm{HC}$ was explained by genetic differences. Heritability estimates were greater than $80 \%$ from young infants to adolescence (18). However, environmental factors, such as maternal level of education, home environmental stimulation, and type of school attended, plus birth $\mathrm{HC}$ and growth in head size in the first $6 \mathrm{mo}$, have been described by many authors as significant determinants for cognition (4). Recent findings from 3,383 children reported that, in the first 6 mo of life, those whose mothers had a low education level had a smaller HC than those from mothers with a high educational level; educational inequalities in HC can be mainly explained by pregnancy-related factors, such as birth weight and gestational age (27).

Inequality between and within populations has origins in adverse early experiences. Developmental neuroscience shows how early biological and psychosocial experiences affect brain development. Cognitive stimulation, stunting, iodine deficiency, and iron-deficiency anemia are risks that prevent millions of young children from attaining their full developmental potential. Recent research emphasizes the importance of these risks, strengthens the evidence for other risk factors including intrauterine growth restriction, malaria, lead exposure, human immunodeficiency virus infection, maternal depression, institutionalization, and exposure to societal violence, and identifies protective factors such as breastfeeding, maternal education, and quality of family care (28).
The findings of this study confirm our hypothesis that independently of grade, school-aged children with the highest scores in the SIMCE tests for both language and mathematics have the highest $\mathrm{HC}$ values compared with their peers with medium and low SA and that, in consequence, $\mathrm{HC}$ is the most relevant anthropometric parameter associated with SA scores. Although the growth of $\mathrm{HC}$ during infancy is known to predict later childhood outcomes, the mechanisms underlying this association with later cognitive abilities remain undetermined. A limitation of this study is that it is very difficult to come to know in detail the biological and psychosocial experiences affecting HC and brain development at an early age. The first $2 \mathrm{y}$ of life represent the period of maximum HC growth, and $70 \%$ of adult brain weight has been attained by the end of the first year (25); furthermore, by the age of $6 y, 90 \%$ of the $\mathrm{HC}$ has been reached (29-32).

Our previous studies on Chilean school-aged children graduating from high school confirmed a high degree of correlation $(r=0.847 ; P<0.0001)$ between absolute $\mathrm{HC}$ and BV measured by magnetic resonance imaging (3); as a consequence, male students with $Z$ - $\mathrm{HC}<-2 \mathrm{SD}$ had a BV $449.1 \mathrm{~cm}^{3}$ lower than the $>2$ SD group, whereas among females, this difference was $214.4 \mathrm{~cm}^{3}(P<0.001)(3)$. This is in accordance with other studies in neonates confirming that $\mathrm{HC}$ is a powerful predictor of total BV. Moreover, BV has been described as a determinant of $\mathrm{HC}$ at term. Microcephaly is associated with lower BVs, 


\section{Articles | Ivanovic et al.}

Table 2. Logistic regression between language and mathematics SAs in the SIMCE test (dependent variable), probability-modeled scores $\geq$ Md, and anthropometric parameters (independent variables), and sex

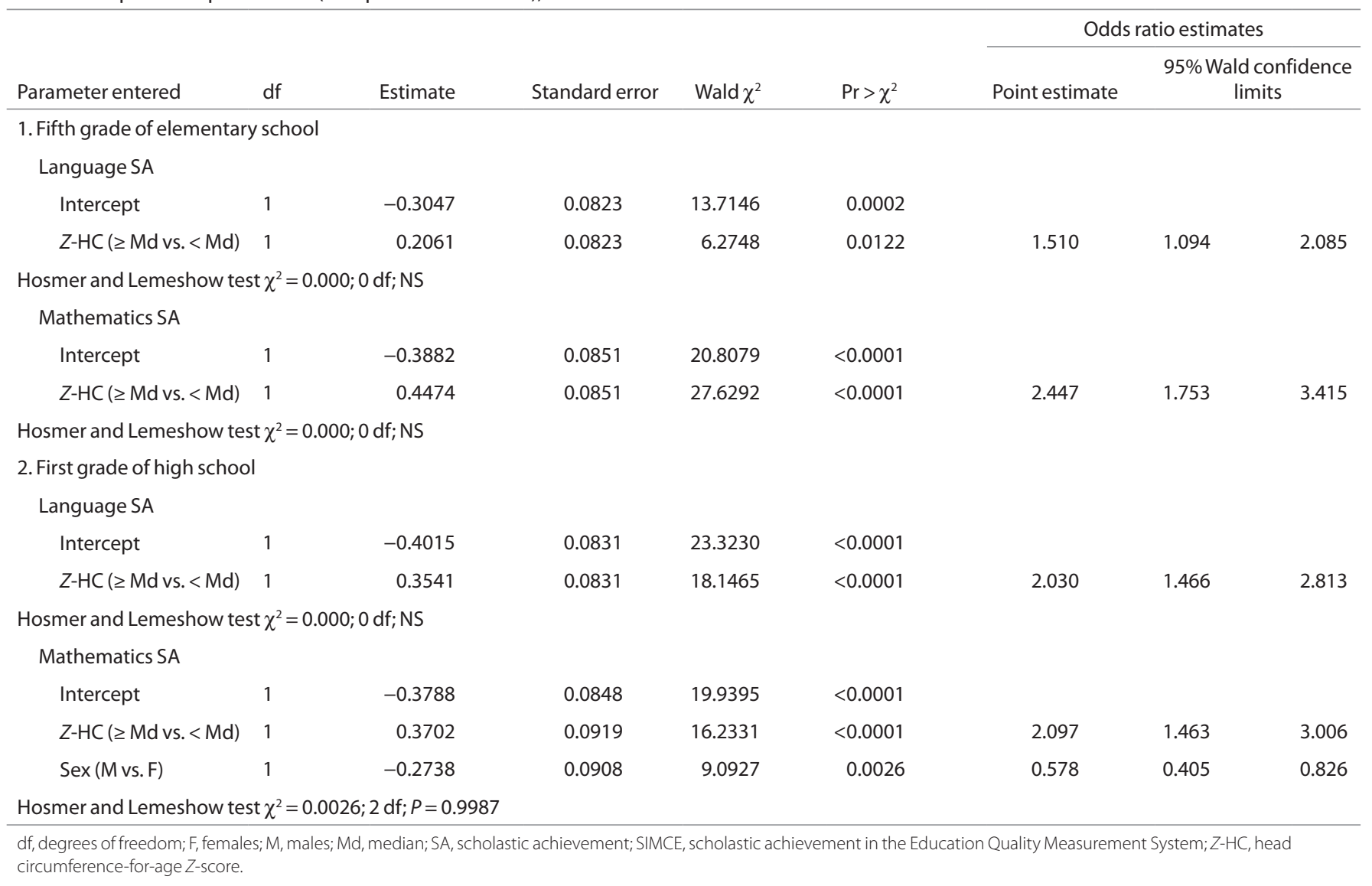

which suggests a vulnerability to adverse factors during gestation. Poor postnatal head growth in preterm infants becomes more evident by $2 \mathrm{y}$ of age and is strongly associated with poor neurodevelopmental outcomes (33).

Our results may provide the stimulus for further research related to the impact of genetic and environmental factors on the HC-SA interrelationship, such as environmental, maternal, home, or school stimulation at an early age. The results of this study clearly demonstrate that low HCs are related to poor SA and children with low HC constitute a vulnerable group for SA. These findings raise important public health questions and provide justification for additional research. The design of the current study does not answer how the low HC relates to poor $\mathrm{SA}$, whether this association may be genetic (in the sense of heritability of smaller $\mathrm{HC}$ and/or heritability of lowered parental intelligence quotient) or due to prenatal nutritional care, postnatal head growth, or maternal stimulation. The importance of our findings could be the justification for a follow-up study that prospectively identifies a birth cohort with small $\mathrm{HC}$ as well as a separate cohort with a small $\mathrm{HC}$ affecting later on in infancy and tracking these children by their growth and development along time. In these children with small $\mathrm{HC}$, it is of interest to determine who are at risk for diminished SA in terms of genetic/heritability factors vs. health/nutritional and environmental stimulation in a multifactorial approach studying their parents and siblings as well. This is a very complicated design but well worth exploring if there is the possibility that earlier intervention could improve SA.

\section{METHODS}

\section{Study Population}

The target population, 187,860 children (39\% of Chilean school population), included all school-aged children enrolled in the fifth elementary school grade $(N=91,663)$ and in the first grade of high school $(N$ $=96,197)$ in Chile's Metropolitan Region in 2010 and who took the SIMCE test at the end of the year 2009. They belonged to public, private subsidized, and private nonsubsidized schools from urban areas.

\section{Sample Selection}

The sampling frame of this study corresponds to the educational establishments from the urban area of the Chilean metropolitan region. The sampling system performed in two stages. In the first stage, 33 educational establishments, which represent $2.61 \%$ of the total population of urban schools $(N=1,262)$, were randomly selected by proportional allocation according to stratification by type of school and the levels of achievement in the SIMCE 2009 established by the Ministry of Education (high, medium, and low). In a second stage, in each of the 33 schools, all students enrolled in both grades who took the SIMCE 2009 were invited to participate in the study. A total of 1,353 school-aged children and their parents agreed to participate in the study and they signed the informed consent form. Their age ranged from 9.9 to $18.2 \mathrm{y}$ (mean age: $10.8 \pm 0.6 \mathrm{y}$ ) and from 12.7 to $17.6 \mathrm{y}$ (mean age: $14.8 \pm 0.6 \mathrm{y}$ ), in the fifth grade of elementary school and in the first year of high school, respectively.

This study was approved by the Committee on Ethics in Studies in Humans of the Institute of Nutrition and Food Technology (INTA), University of Chile, and ratified by the Committee on Bioethics of the National Fund for Scientific and Technologic Development 
(FONDECYT), Chile. Subjects' consent was obtained according to the norms for Human Experimentation, Code of Ethics of the World Medical Association (Declaration of Helsinki) (34). The field study was carried out during 2010.

\section{SIMCE Tests}

SA was assessed through the SIMCE tests, which has national coverage in Chile and is administered by the Ministry of Education; this was considered the dependent variable. The aim of this testing is to improve the quality and equitableness of the educational process in the different areas covered by the national curriculum. Scores range between 0 and 400, and the results are expressed as scores (mean \pm $\mathrm{SD}$ ) in the language and mathematics tests. Data were also categorized in three ranges according to the levels of achievement established by the Ministry of Education of Chile for both grades. In grade IV, results for language scores in the 2009 SIMCE test were classified as follows: high SA (score $\geq 281$ ), medium SA (score 241-280), and low SA (score $\leq 240$ ); and for mathematics scores, high SA (score $\geq$ 286), medium SA (score 233-285), and low SA (score $\leq 232$ ). In grade VIII, results for language scores in the 2009 SIMCE test were classified as follows: high SA (score $\geq 286$ ), medium SA (score 235-285), and low SA (score $\leq 234$ ); for the mathematics scores, the high SA was score $\geq 321$, for medium levels, the SA score was $276-320$, and for the low level, the SA was score $\leq 275$ (35).

\section{Anthropometric Measurements}

The measurements of weight, height, and $\mathrm{HC}$ were carried out at school applying standardized procedures; all the instruments were verified before measuring each subject (36). Nutritional status was expressed as $Z-\mathrm{H}$ and classified as severe growth failure $(Z$-score $<-2$ $\mathrm{SD})$, mild growth failure ( $-2 \mathrm{SD}$ to $<-1 \mathrm{SD})$, normal height $(Z$-score between -1 and $1 \mathrm{SD}$ ), and tall ( $Z$-score $>1 \mathrm{SD}$ ) (37). Their BMI (evaluated as weight/height ${ }^{2}$ ) was compared with National Center for Health Statistics-Centers for Disease Control and Prevention tables, and children were distributed into four categories: underweight ( $Z$-score $<-1 \mathrm{SD})$, well nourished ( $Z$-score between -1 and $1 \mathrm{SD}$ ), obesity risk ( $Z$-score between +1 and $+2 \mathrm{SD})$, and obesity ( $Z$-score $>+2$ SD) (37). BMI was calculated using biological age derived from Tanner stages (29). Raw HC was compared with the tables of Tanner (29), Nellhaus (30), Roche et al. (31), and Ivanovic et al. (32) and was expressed as $Z$-HC). $Z$-HC values were similar when applying these tables because the correlation coefficient between these patterns was 0.98 (32). In this respect, mean \pm 2 SD was considered "normal HC," $<-2$ SD as microcephaly, and $>+2$ SD as macrocephaly (29-32). In this study, the classification was as follows: $<-2$ SD, $<0$ to -2 SD, 0 to $2 \mathrm{SD}$, and $>2$ SD. The anthropometric parameters were considered independent variables.

\section{Socioeconomic Stratum}

Socioeconomic stratum was measured applying a scale based on Graffar's modified method that had been adapted to Chilean urban and rural populations (38). This scale classified the sample into five socioeconomic strata: $1=$ high $(0.3 \%) ; 2=$ medium to high $(14.7 \%)$; $3=$ medium $(38.3 \%) ; 4$ = medium to low (45.6\%); and $5=$ low $(1.1 \%)$.

\section{Statistical Analysis}

Data were processed using the Statistical Analysis System package (SAS 9.3; SAS Institute (Cary, NC)). Statistical analysis included $\chi^{2}$ for categorical variables, variance tests, and Bonferroni test for comparison of means. Logistic regression with the option "Stepwise" was used to establish the most important independent variables that affect language and mathematics SA (dependent variable). Language and mathematics scores were separately considered dependent variables and divided into two groups: $<\mathrm{Md}$ and $\geq \mathrm{Md}$. Probability modeled was language and mathematics scores $\geq \mathrm{Md}$. Hosmer and Lemeshow test was applied for goodness of fit. The independent variables considered in the statistical model were the anthropometric parameters significantly associated with SA, $Z$-H (with growth failure and without growth failure), and $Z$-HC ( $<0 \mathrm{SD}$ and $\geq 0 \mathrm{SD}$ ), controlling for sex (39).

\section{ACKNOWLEDGMENTS}

The authors are very grateful to the Ministry of Education of Chile for all the facilities given to carry out this research; to Oscar Brunser, for helpful comments and suggestions; and to Leopoldo Salgado, for their photography work. The data in this manuscript are part of a thesis submitted by the second author C.V.I., in partial fulfillment of the requirements for the Master of Nutritional Sciences degree from the Institute of Nutrition and Food Technology (INTA), University of Chile.

This study was presented in part at the 52nd Annual Meeting of the European Society for Paediatric Research (ESPR 2011) (Pediatric Research (2011) 70, 326).

\section{STATEMENT OF FINANCIAL SUPPORT}

This study was supported in part by Grant 1100431 from the National Fund for Scientific and Technologic Development (FONDECYT), Santiago, Chile.

Disclosure: The authors declared no conflict of interest.

\section{REFERENCES}

1. Gale CR, O'Callaghan FJ, Bredow M, Martyn CN; Avon Longitudinal Study of Parents and Children Study Team. The influence of head growth in fetal life, infancy, and childhood on intelligence at the ages of 4 and 8 years. Pediatrics 2006;118:1486-92.

2. Ochiai M, Nakayama H, Sato K, et al. Head circumference and long-term outcome in small-for-gestational age infants. J Perinat Med 2008;36:341-7.

3. Ivanovic DM, Leiva BP, Pérez HT, et al. Head size and intelligence, learning, nutritional status and brain development. Head, IQ, learning, nutrition and brain. Neuropsychologia 2004;42:1118-31.

4. Emond AM, Lira PI, Lima MC, Grantham-McGregor SM, Ashworth A. Development and behaviour of low-birthweight term infants at 8 years in northeast Brazil: a longitudinal study. Acta Paediatr 2006;95:1249-57.

5. Frisk V, Amsel R, Whyte HE. The importance of head growth patterns in predicting the cognitive abilities and literacy skills of small-for-gestationalage children. Dev Neuropsychol 2002;22:565-93.

6. Johnson S, Wolke D, Hennessy E, Marlow N. Educational outcomes in extremely preterm children: neuropsychological correlates and predictors of attainment. Dev Neuropsychol 2011;36:74-95.

7. Silva LM, Jansen PW, Steegers EA, et al. Mother's educational level and fetal growth: the genesis of health inequalities. Int J Epidemiol 2010;39:1250-61.

8. Cornelius MD, Goldschmidt L, Willford JA, Leech SL, Larkby C, Day NL. Body size and intelligence in 6-year-olds: are offspring of teenage mothers at risk? Matern Child Health J 2009;13:847-56.

9. Charkaluk ML, Truffert P, Marchand-Martin L, et al.; Epipage study group. Very preterm children free of disability or delay at age 2: predictors of schooling at age 8: a population-based longitudinal study. Early Hum Dev 2011;87:297-302.

10. Vieira ME, Linhares MB. Developmental outcomes and quality of life in children born preterm at preschool- and school-age. J Pediatr (Rio J) 2011;87:281-91.

11. Lorenz JM, Whitaker AH, Feldman JF, et al. Indices of body and brain size at birth and at the age of 2 years: relations to cognitive outcome at the age of 16 years in low birth weight infants. J Dev Behav Pediatr 2009;30:535-43.

12. Abernethy LJ, Cooke RW, Foulder-Hughes L. Caudate and hippocampal volumes, intelligence, and motor impairment in 7-year-old children who were born preterm. Pediatr Res 2004;55:884-93.

13. Northam GB, Liégeois F, Chong WK, Wyatt JS, Baldeweg T. Total brain white matter is a major determinant of IQ in adolescents born preterm. Ann Neurol 2011;69:702-11.

14. Isaacs EB, Fischl BR, Quinn BT, Chong WK, Gadian DG, Lucas A. Impact of breast milk on intelligence quotient, brain size, and white matter development. Pediatr Res 2010;67:357-62.

15. Isaacs EB, Gadian DG, Sabatini S, et al. The effect of early human diet on caudate volumes and IQ. Pediatr Res 2008;63:308-14.

16. Pangelinan MM, Zhang G, VanMeter JW, Clark JE, Hatfield BD, Haufler AJ. Beyond age and gender: relationships between cortical and subcortical brain volume and cognitive-motor abilities in school-age children. Neuroimage 2011;54:3093-100.

17. McDaniel MA. Big-brained people are smarter: a meta-analysis of the relationship between in vivo brain volume and intelligence. Intelligence 2005;33:337-46. 


\section{Articles | Ivanovic et al.}

18. Smit DJ, Luciano M, Bartels M, et al. Heritability of head size in Dutch and Australian twin families at ages 0-50 years. Twin Res Hum Genet 2010;13:370-80.

19. Ivanovic DM, Pérez HT, Olivares MG, Díaz NS, Leyton BD, Ivanovic RM. Scholastic achievement: a multivariate analysis of nutritional, intellectual, socioeconomic, sociocultural, familial, and demographic variables in Chilean school-age children. Nutrition 2004;20:878-89.

20. Ivanovic R, Forno H, Castro CG, Ivanovic D. Intellectual ability and nutritional status assessed through anthropometric measurements of Chilean school-age children from different socioeconomic status. Ecol Food Nutr 2000;39:35-59.

21. Ivanovic D, Del P Rodríguez M, Pérez H, et al. Twelve-year follow-up study of the impact of nutritional status at the onset of elementary school on later educational situation of Chilean school-age children. Eur J Clin Nutr 2008;62:18-31.

22. Ivanovic DM, Rodríguez M del P, Pérez $\mathrm{H}$, et al. Long-term study of the impact of nutritional, intellectual, family, educational and socio-economic variables of school-age children at the onset of elementary school on the academic aptitude test achievement at the end of high school. Chile's Metropolitan Region. 1987-1998. Br J Nutr 2009;102:142-54.

23. Székely B. Los Tests, vol. 1, 5a Edición. Buenos Aires, Argentina: Editorial Kapeluz, 1966.

24. Mannerkoski MK, Heiskala HJ, Van Leemput K, et al. Subjects with intellectual disability and familial need for full-time special education show regional brain alterations: a voxel-based morphometry study. Pediatr Res 2009;66:306-11.

25. Ivanovic DM, Leiva BP, Perez HT, et al. Long-term effects of severe undernutrition during the first year of life on brain development and learning in Chilean high-school graduates. Nutrition 2000;16:1056-63.

26. Ramakrishnan U, Stein AD, Parra-Cabrera S, et al. Effects of docosahexaenoic acid supplementation during pregnancy on gestational age and size at birth: randomized, double-blind, placebo-controlled trial in Mexico. Food Nutr Bull 2010;31(Suppl):S108-16.

27. Bouthoorn SH, van Lenthe FJ, Hokken-Koelega AC, et al. Head circumference of infants born to mothers with different educational levels; the Generation R Study. PLoS ONE 2012;7:e39798.
28. Walker SP, Wachs TD, Grantham-McGregor S, et al. Inequality in early childhood: risk and protective factors for early child development. Lancet 2011;378:1325-38.

29. Tanner JM. Physical growth and development. In: Forfar JO, Arneil GC, eds. Textbook of Pediatrics. Edinburgh, Scotland: Churchill Livingstone, 1984:278-330.

30. Nellhaus G. Head circumference from birth to eighteen years. Practical composite international and interracial graphs. Pediatrics 1968;41:10614.

31. Roche AF, Mukherjee D, Guo SM, Moore WM. Head circumference reference data: birth to 18 years. Pediatrics 1987;79:706-12.

32. Ivanovic D, Olivares M, Castro C, Ivanovic R. [Head circumference in Chilean school children 5 to 18 years of age and socioeconomic status]. Rev Med Chil 1995;123:587-99.

33. Cheong JL, Hunt RW, Anderson PJ, et al. Head growth in preterm infants: correlation with magnetic resonance imaging and neurodevelopmental outcome. Pediatrics 2008;121:e1534-40.

34. The World Medical Association. Declaration of Helsinki-Ethical Principles for Medical Research Involving Human Subjects (Declaration of Helsinki). 59th WMA General Assembly, Seoul, Korea, October 2008.

35. Chile. Ministerio de Educación. Resultados Nacionales SIMCE 2009 [SIMCE 2009 National Results]. SIMCE, Unidad de Currículum y Evaluación (UCE). Santiago: Ministerio de Educación, 2010.

36. Gibson R. Principles of Nutritional Assessment. Oxford, UK: Oxford University Press, 1990.

37. National Center for Health Statistics. Growth curves. CDC growth Charts for United States: Methods and development, 2000 (www.cdc.gov/growthcharts.).

38. Alvarez ML, Muzzo S, Ivanovic D. [Scale for measurement of socioeconomic level, in the health area]. Rev Med Chil 1985;113:243-9.

39. Guilford JP, Fruchter B. Estadística Aplicada a la Psicología y a la Educación [Fundamental statistics in psychology and education]. México: McGraw Hill, 1984. 